\title{
BDNF Val66Met is Associated with Introversion and Interacts with 5-HTTLPR to Influence Neuroticism
}

\author{
Antonio Terracciano*,', Toshiko Tanaka', Angelina R Sutin', Barbara Deiana ${ }^{2}$, Lenuta Balaci², \\ Serena Sanna ${ }^{2}$, Nazario Olla ${ }^{2}$, Andrea Maschio ${ }^{2}$, Manuela Uda ${ }^{2}$, Luigi Ferrucci', David Schlessinger' \\ and Paul T Costa Jr' \\ 'National Institute on Aging, NIH, DHHS, Baltimore, MD, USA; ${ }^{2}$ Istituto di Neurogenetica e Neurofarmacologia, Consiglio Nazionale delle \\ Ricerche, Monserrato, Cagliari, Italy
}

\begin{abstract}
Brain-derived neurotrophic factor (BDNF) regulates synaptic plasticity and neurotransmission, and has been linked to neuroticism, a major risk factor for psychiatric disorders. A recent genome-wide association (GWA) scan, however, found the BDNF Val66Met polymorphism (rs6265) associated with extraversion but not with neuroticism. In this study, we examine the links between BDNF and personality traits, assessed using the Revised NEO Personality Inventory (NEO-PI-R), in a sample from SardiNIA $(n=1560)$ and the Baltimore Longitudinal Study of Aging (BLSA; $n=|1| 3 \mid$ ). Consistent with GWA results, we found that BDNF Met carriers were more introverted. By contrast, in both samples and in a meta-analysis inclusive of published data $(n=|525|)$, we found no evidence for a main effect of BDNF Val66Met on neuroticism. Finally, on the basis of recent reports of an epistatic effect between BDNF and the serotonin transporter, we explored a Val66Met $\times 5$-HTTLPR interaction in a larger SardiNIA sample $(n=2333)$. We found that 5-HTTLPR LL carriers scored lower on neuroticism in the presence of the BDNF Val variant, but scored higher on neuroticism in the presence of the BDNF Met variant. Our findings support the association between the BDNF Met variant and introversion and suggest that BDNF interacts with the serotonin transporter gene to influence neuroticism.

Neuropsychopharmacology (2010) 35, 1083-1089; doi:I0.1038/npp.2009.213; published online 30 December 2009
\end{abstract}

Keywords: personality; depression; BDNF Val66Met; serotonin transporter; GWA; gene-gene interaction.

\section{INTRODUCTION}

Brain-derived neurotrophic factor (BDNF) is highly expressed in the brain and is implicated in several neural processes, including neurogenesis, differentiation, survival, and synaptic plasticity. Animal models and human studies indicate that BDNF secretion is activity dependent: Cognitive stimulation, physical activity, and antidepressant treatment increase BDNF secretion, whereas stress and mood disorders decrease it (Chen et al, 2001; Cotman and Berchtold, 2002; Martinowich and Lu, 2008; Mattson et al, 2004; Sen et al, 2008). There is also considerable evidence that the genetic variant Val66Met (rs6265) influences the BDNF system. This single-nucleotide polymorphism (SNP) results in a Val to Met substitution. The less-frequent Met variant has been associated with impaired memory and hippocampal function (Egan et al, 2003; Matsuo et al, 2009), smaller hippocampal volume (Bueller et al, 2006), and, in

*Correspondence: Dr A Terracciano, Laboratory of Personality and Cognition, National Institute on Aging, NIH, DHHS, 25I Bayview Blvd, Baltimore, MD 21224, USA, Tel: + | 410558 8358, Fax: + | 410558 8690, E-mail: terraccianoa@mail.nih.gov

Received 27 July 2009; revised 9 November 2009; accepted 20 November 2009 transgenic knock-in mice, defective BDNF secretion and increased anxiety-related behaviors (Chen et al, 2006).

The BDNF Val66Met has been studied extensively as a candidate gene for mood disorders and schizophrenia, but the results are mixed (Gratacos et al, 2007; Green et al, 2006; Muglia et al, 2008; Rybakowski, 2008; Sklar et al, 2002). Several studies (Hunnerkopf et al, 2007; Itoh et al, 2004; Lang et al, 2005; Sen et al, 2003; Tochigi et al, 2006; WillisOwen et al, 2005a; Wray et al, 2008) have also examined the association between BDNF and the personality trait neuroticism, a major risk factor for depression, anxiety, and other psychiatric disorders (Bienvenu et al, 2004; Clark et al, 1994; Jylha et al, 2009; Kendler et al, 2006; Watson et al, 2005). A recent meta-analysis concluded that the lessfrequent Met variant is associated with lower neuroticism (Frustaci et al, 2008), an unexpected finding, given the unfavorable effects of the Met variant (eg, abnormal hippocampal structure and function). However, a large study (Willis-Owen et al, 2005a) and two recent genomewide association (GWA) scans (Terracciano et al, 2008; van den Oord et al, 2008) found no association between Val66Met and neuroticism. In light of these results, it remains an open question whether Val66Met has a direct effect on neuroticism. 
Interestingly, the only published GWA study of extraversion reported that Met carriers were more introverted (Terracciano et al, 2008). This association was not seen in previous studies (Itoh et al, 2004; Sen et al, 2003; Tochigi et al, 2006), perhaps because of their small sample sizes $(N<450)$. Owing to its relation to emotional processes, an association between BDNF and extraversion is of considerable interest. Extraversion is inversely and weakly associated with the experience of negative affect, but has a pervasive effect on positive emotions and life satisfaction (Costa and McCrae, 1980; Watson and Clark, 1992). Introverts are more likely to experience anhedonia and dysthymic/depressed mood (Clark et al, 1994; Jylha et al, 2009; Watson et al, 2005), important clinical features of depressive disorders.

The present research has two major goals. The first goal is to further test the association between BDNF Val66Met and both neuroticism and extraversion in samples from the United States and Italy. To provide a quantitative summary of the association between BDNF and neuroticism, we conducted a meta-analysis combining this study with the published literature.

The second goal of this study was to test the hypothesis that BDNF Val66Met and a polymorphism in the promoter region of the serotonin transporter gene (5-HTTLPR) interact to influence neuroticism scores. This hypothesis derives from biological evidence that BDNF functions as a synaptic modulator that regulates multiple neurotransmitter systems, including serotonin, dopamine, and glutamate (Carvalho et al, 2008; Hunnerkopf et al, 2007; Mossner et al, 2000). In particular, BDNF stimulates the sprouting and morphological differentiation of serotonin neurons and enhances their survival (Mamounas et al, 1995; Martinowich and $\mathrm{Lu}, 2008$; Mattson et al, 2004). Infusion of BDNF into the brain increases activity in monoaminergic systems, whereas depletion of serotonin downregulates BDNF mRNA (Martinowich and Lu, 2008; Zhou et al, 2008). The interdependence of the BDNF and serotonin systems is also supported by findings that BDNF has antidepressant-like effects (Siuciak et al, 1996). Chronic antidepressant treatment, including selective serotonin reuptake inhibitors (SSRIs), upregulates BDNF expression (Nibuya et al, 1995).

The interaction of serotonin and BDNF systems has generated hypotheses of genetic epistasis, and recent studies have reported interactions between BDNF Val66Met and 5HTTLPR. In these studies, the outcomes of epistatic effects were measures of depressive symptoms in the presence of environmental adversity (Kim et al, 2007; Wichers et al, 2008), differences in mood-related brain structures (Pezawas et al, 2008), or response to mood stabilizers (Rybakowski et al, 2007). In this study, we examine the interaction between the serotonin transporter and BDNF genes in the largest sample tested so far on a depressionrelated phenotype.

\section{SUBJECTS AND METHODS}

\section{Sample Description: SardiNIA}

SardiNIA is a study conducted in Sardinia, Italy, supported by the National Institute on Aging (NIA). The study has been described in detail elsewhere (Costa et al, 2007; Pilia et al, 2006; Terracciano et al, 2008; Terracciano et al, $2009 \mathrm{~b})$. We recruited about $62 \%$ of the population aged $14-$ 102 years $(n=6148)$ from a cluster of four towns in the Lanusei Valley, Sardinia. As part of a founder population, the recruited individuals are highly interrelated and most have common ancestors from the same isolated region. Valid personality data were obtained from 5693 subjects at their first assessment, of which 3972 were genotyped as part of a GWA study (Terracciano et al, 2008). Here, we report association analyses for 1560 individuals who are a part of the SardiNIA cohort, but were not included in the original GWA analyses (Terracciano et al, 2008). We refer to this sample as SardiNIA 2. In this sample, age ranged from 14 to 94 years $(M=42.7 ; S D=17)$, with $59.9 \%$ women.

To examine the interaction between BDNF Val66Met and 5-HTTLPR, we combined the two SardiNIA samples. We included individuals who were genotyped for both polymorphisms (as part of the GWA study or SardiNIA 2) and assessed for personality traits $(n=2333)$. In this sample, age ranged from 14 to 94 years $(M=45.8 ; S D=17)$, with $57 \%$ women. The SardiNIA project was approved by institutional review boards in Italy and the United States, and research was carried out in accordance with the Declaration of Helsinki Principles and other international guidelines.

\section{Sample Description: BLSA}

The Baltimore Longitudinal Study of Aging (BLSA) is an ongoing multidisciplinary study of community-dwelling volunteers. Here, we examine data from 1131 subjects who were successfully genotyped for Val66Met and completed the personality questionnaire at least once. In this sample, age at first assessment ranged from 20 to 93 years $(\mathrm{M}=57.3$; $\mathrm{SD}=15.5$ ), with $48 \%$ women. About $74 \%$ of the participants were of European descent, 25\% African American, and 1\% were Asian American. Personality traits were assessed from 1989 to 2009, and multiple assessments were available for $86 \%$ of the participants. Although personality traits are generally stable over time (Terracciano et al, 2006, in press), to provide more robust estimates, we used the average across all available assessments. These average scores are likely to provide a more reliable estimate of the individuals' personality profile. When multiple assessments were available, average age was used as covariate. The sample included a similar number of men and women with multiple assessments. The BLSA study was approved by the local institutional review board, and research was carried out in accordance with the Declaration of Helsinki Principles and other international guidelines.

\section{Personality Assessment}

Personality traits were assessed using the English (Costa and McCrae, 1992) and Italian (Terracciano, 2003) versions of the Revised NEO Personality Inventory (NEO-PI-R). The questionnaire consists of 240 items answered on a 5-point Likert scale, from strongly disagree to strongly agree. Scores followed a normal distribution and were standardized $(\mathrm{M}=50, \mathrm{SD}=10)$ using American combined gender norms (Costa and McCrae, 1992).

The NEO-PI-R has a robust factor structure that has been replicated in Italy (Terracciano, 2003) and in more than 50 
cultures (McCrae and Terracciano, 2005a), even at the genetic level (Costa et al, 2007; Pilia et al, 2006). In both samples, the NEO-PI-R showed good psychometric properties: internal consistency reliabilities for the five factors were at or above 0.80 . In the BLSA, available longitudinal data indicate that stability coefficients for the five factors are in the range of 0.80 over intervals of 10 years (Terracciano et al, 2006). In addition, twin studies have shown that both neuroticism and extraversion are heritable, suggesting a genetic basis for these two traits (Bouchard and Loehlin, 2001). In the SardiNIA sample, family-based estimates of broad heritability were 0.44 for neuroticism and 0.39 for extraversion (Pilia et al, 2006).

\section{Genetic Assays}

The BDNF SNP rs6265 was genotyped using an Affymetrix custom chip in the SardiNIA 2 sample, and with the 550k Illumina platform in the BLSA sample. Genotype data from both samples passed quality controls, including HardyWeinberg equilibrium. In the SardiNIA 2 sample, genotype frequencies were $45.3 \%$ for $\mathrm{GG}(\mathrm{Val} / \mathrm{Val}), 44.5 \%$ for $\mathrm{AG}$ (Val/Met), and $10.2 \%$ for AA (Met/Met). The frequency of the G (Val) allele was $68 \%$, which is lower than the frequencies found in other European populations $(\sim 81 \%)$. In the BLSA sample, genotype frequencies were $68.1 \%$ for GG (Val/Val), $28.6 \%$ for AG (Val/Met), and $3.3 \%$ for $\mathrm{AA}$ (Met/Met). The frequency of the $\mathrm{G}(\mathrm{Val})$ allele was $82 \%$.

As described elsewhere (Terracciano et al, 2009a), in the SardiNIA sample, 5-HTTLPR genotypes were determined using the MegaBACE 1000 fluorescence-based genotyping methodology. PCR was successful in $>95 \%$ of the samples, and there were no assay-specific problems with the MegaBACE 1000 automatic DNA sequencer. In the samples that passed quality controls, allele frequencies were $49 \%$ for the short (S) allele and $51 \%$ for the long (L) allele, and genotype frequencies were in Hardy-Weinberg equilibrium $(P>0.05)$. Some evidence suggests that the effect of the 5-HTTLPR genotype on the serotonin transporter gene expression is further influenced by a polymorphism of the 5-HTTLPR long allele (rs25531), but we did not genotype this variant. However, several studies that genotyped the rs25531 polymorphism have found no association with neuroticism, anxiety, depression, or obsessive compulsive disorder (Gunthert et al, 2007; Wachleski et al, 2008; Wendland et al, 2007; Wray et al, 2009).

\section{Statistical Analyses}

To take advantage of the family information in the SardiNIA sample, we used the program MERLIN (Chen and Abecasis, 2007) to perform association analyses between Val66Met and personality traits. In MERLIN, regression coefficients are estimated in the context of a variance component model to account for relatedness among individuals (Chen and Abecasis, 2007). The same analyses were carried out for BLSA. Sex, age, and age ${ }^{2}$ were included as covariates in all analyses to account for sex differences and the linear (age) and curvilinear $\left(\mathrm{age}^{2}\right)$ effects of age on personality traits (Costa et al, 2001; Terracciano et al, 2005).

Although it is difficult to account for the family structure and more complex genetic effects in power calculations, on the basis of a simulation of statistical power performed elsewhere (Willis-Owen et al, 2005a), both samples should have adequate power $(>80 \%)$ to detect the main genetic effect observed in the original SardiNIA GWA study.

In the SardiNIA sample, we checked the genomic control value for our GWA analyses (Devlin and Roeder, 1999), and carried out a principal component analysis of genome-wide SNP data in a subset of unrelated individuals (Price et al, 2006). Neither analysis suggested evidence of population substructure nor did genetic outliers in this sample. To account for the multiethnic composition of the BLSA sample, we included eigenvalues derived from the full GWA data set (Price et al, 2006) as covariates and we repeated the analyses restricting them to the EuropeanAmerican subsample.

Regression analyses were carried out to examine the interaction effect (SPSS 13.0). A first set of analyses examined interactions of all Val66Met (Val/Val, Val/Met, and Met/Met) and 5-HTTLPR (SS, SL, and LL) genotypes. Following previous studies (Pezawas et al, 2008; Rybakowski et al, 2007; Terracciano et al, 2009a), we also contrasted the $\mathrm{Val} / \mathrm{Val}$ genotype with any-Met genotypes, and LL with any$S$ genotypes. The regressions for the three- and two-way interactions controlled for sex, age, and $\mathrm{age}^{2}$. Interaction effects were further evaluated by MERLIN association analyses of BDNF with neuroticism separately for the 5-HTTLPR LL and any-S genotype groups.

\section{Meta-Analysis}

A recent meta-analysis reported an association between neuroticism and the BDNF Val66Met variant (Frustaci et al, 2008). We update this meta-analysis by adding data from the samples in this study and from the previous GWA study (Terracciano et al, 2008). We searched (up to 20 July 2009) for additional articles through the PubMed and Scopus search engines using the following keywords: 'Neuroticism,' 'BDNF,' 'brain derived neurotrophic factor,' 'rs6265,' and 'Val66Met.' We also examined references and citations of relevant studies. We included all samples genotyped for Val66Met and assessed with a measure of neuroticism. Surprisingly, the largest study we found (Willis-Owen et al, 2005a) was published in 2005, but was not included in the 2008 meta-analysis (Frustaci et al, 2008). We carried out the meta-analysis, examining fixed and random effect models, using the program Comprehensive Meta Analysis (version 2). In line with previous studies (Gatt et al, 2009; Pezawas et al, 2008; Rybakowski et al, 2007; Sen et al, 2003), given the small number of Met/Met genotypes among European populations, we contrasted the standardized mean difference on neuroticism between the $\mathrm{Val} / \mathrm{Val}$ genotype $v s$ the combined $\mathrm{Val} / \mathrm{Met}$ and Met/Met genotypes.

\section{RESULTS}

Table 1 presents neuroticism and extraversion scores by the BDNF Val66Met (rs6265) genotype for the SardiNIA and BLSA samples. Consistent with the results of the GWA scan (Terracciano et al, 2008), individuals with the BDNF Met variant scored significantly lower on extraversion in both the SardiNIA (allele: G; $\beta=0.093 ; p=0.005$ ) and BLSA 
(allele: $\mathrm{G} ; \beta=0.114 ; p=0.026)$ samples. The association was slightly stronger (allele: $\mathrm{G} ; \beta=0.153 ; p=0.008$ ) in the BLSA sample when analyses were restricted to participants of European ancestry $(n=839)$. In both samples, the Val/Met group tends to score roughly $0.2 \mathrm{SD}$ lower on extraversion compared with the Val/Val group.

We found no association between Val66Met genotypes and neuroticism in either SardiNIA $(p=0.19)$ or BLSA $(p=0.94)$ samples. Because these results are inconsistent with the previous meta-analysis based on 5 studies and 1633 subjects (Frustaci et al, 2008), we carried out a new metaanalysis combining data from the published literature to date and this study, for a total of 13 samples and 15251 subjects. Figure 1 presents a forest plot of standardized mean differences on neuroticism between $\mathrm{Val} / \mathrm{Val}$ and

Table I Neuroticism and Extraversion Scores by BDNF Val66Met Genotypes

\begin{tabular}{lcccc}
\hline & \multicolumn{3}{c}{ BDNF genotype } & \multirow{2}{*}{ P-value } \\
\cline { 2 - 3 } & GG Val/Val & AG Val/Met & AA Met/Met & \\
\hline SardiNIA (N) & 707 & 694 & 159 & \\
Neuroticism & $55.0(9.5)$ & $55.7(9.2)$ & $55.5(8.7)$ & 0.19 \\
Extraversion & $49.6(8.9)$ & $47.5(9.4)$ & $48.9(8.8)$ & 0.005 \\
BLSA (N) & & & & \\
Neuroticism & $46.8(8.3)$ & $48.3(8.6)$ & $44.2(10.2)$ & 0.94 \\
Extraversion & $51.5(9.9)$ & $49.1(9.6)$ & $50.0(10.2)$ & 0.02 \\
& & & & \\
BLSA-Europeans $(N)$ & 515 & 290 & 34 & \\
Neuroticism & $47.4(8.4)$ & $48.4(8.6)$ & $44.8(9.9)$ & 0.74 \\
Extraversion & $50.8(10.2)$ & $48.8(9.9)$ & $49.7(10.3)$ & 0.008 \\
\hline
\end{tabular}

Abbreviations: BDNF, brain-derived neurotrophic factor, BLSA, Baltimore Longitudinal Study of Aging.

Raw personality domain scores (SD). P-values derived from association analyses carried out using MERLIN controlling for sex, age, and age ${ }^{2}$. In the full BLSA sample, eigenvalues were used as additional covariates to control for population stratification.
Val/Met + Met/Met BDNF genotypes. Apart from the few small studies that show trends in either direction, the largest studies show no evidence of an association between neuroticism and BDNF Val66Met. There was no significant heterogeneity $(Q=16.9 ; p>0.05)$, and the pooled effect sizes using the fixed- and random-effects model were 0.01 ( $95 \%$ CI: -0.02 to $0.05 ; p=0.39)$ and 0.02 (95\% CI: -0.03 to $0.06 ; p=0.51)$.

\section{Interaction of BDNF and 5-HTTLPR}

Recent studies have reported interactions between Val66Met and 5-HTTLPR on depression-related phenotypes (Pezawas et al, 2008; Rybakowski et al, 2007; Wichers et al, 2008). We tested for such an interaction on neuroticism, a major risk factor for mood disorders. We first examined the interaction of the SS, SL, and LL 5-HTTLPR genotypes with $\mathrm{Val} / \mathrm{Val}, \mathrm{Val} / \mathrm{Met}$, and Met/Met BDNF variants. Consistent with previous studies, we also repeated the analyses combining the genotype groups with any $S$ allele of the 5-HTTLPR and the genotype groups with any Met variant of BDNF. When the three genotypes of the two genes were examined, the interaction term was not significant. However, when a $2 \times 2$ interaction was tested, we observed a significant effect $(p<0.05)$. Figure 2 presents neuroticism scores stratified by BDNF and 5-HTT variants. Among individuals with LL 5-HTTLPR, BDNF Met carriers scored higher on neuroticism compared with those with the Val/ Val BDNF genotype (allele: G; $\beta=-0.169 ; p=0.005$ ). Among those with the $S$ 5-HTTLPR allele, no differences were observed among BDNF genotypes (allele: G; $\beta=0.00$; $p=0.98$ ). For extraversion, we found no statistically significant interaction between BDNF and 5-HTTLPR $(\beta=-0.029 ; p=0.52)$.

\section{DISCUSSION}

This study tested whether the BDNF Val66Met variant had a main effect on extraversion and neuroticism, and whether the Val66Met variant interacted with 5-HTTLPR to influence neuroticism scores. Consistent with a relatively large GWA study in the SardiNIA cohort (Terracciano et al,
Study name

2003 Sen et al

2004 Itoh et al

2005 Lang et al.

2005 Willis-Owen sibs

2005 Willis-Owen Singletons

2005 Willis-Owen EPIC-Norfolk

2006 Tochigi et al

2007 Hünnerkopf et al

2008 Wray et al

2009 Gattet al

2009 BLSA

2009 SardiNIA1

2009 SardiNIA2

Total

\begin{tabular}{|c|c|c|}
\hline $\mathrm{N}$ & SMD & $\mathrm{p}$-value \\
\hline 436 & 0.21 & 0.0 \\
\hline 151 & -0.01 & \\
\hline 343 & 0.19 & \\
\hline 516 & -0.13 & 0. \\
\hline 571 & -0.11 & \\
\hline 4843 & 0.02 & \\
\hline 424 & 0.14 & \\
\hline 272 & 0.01 & \\
\hline 950 & 0.02 & \\
\hline 374 & 0.11 & \\
\hline 839 & -0.01 & \\
\hline 3972 & 0.03 & 0 \\
\hline 1560 & -0.08 & \\
\hline 5251 & 0.01 & \\
\hline
\end{tabular}

0.39
SMD and $95 \% \mathrm{Cl}$

0.03

0.09

0.16

0.20

0.56

0.16

0.82

0.30

0.83

0.34

.14

-0.50 Any Met $0.00 \quad \mathrm{Val} / \mathrm{Val}$

Figure I Forest plot of studies examining the association between neuroticism and the BDNF Val66Met polymorphism. Met/Met and Val/Met were contrasted with the $\mathrm{Val} / \mathrm{Val}$ genotypes. SMD, standardized mean differences. 

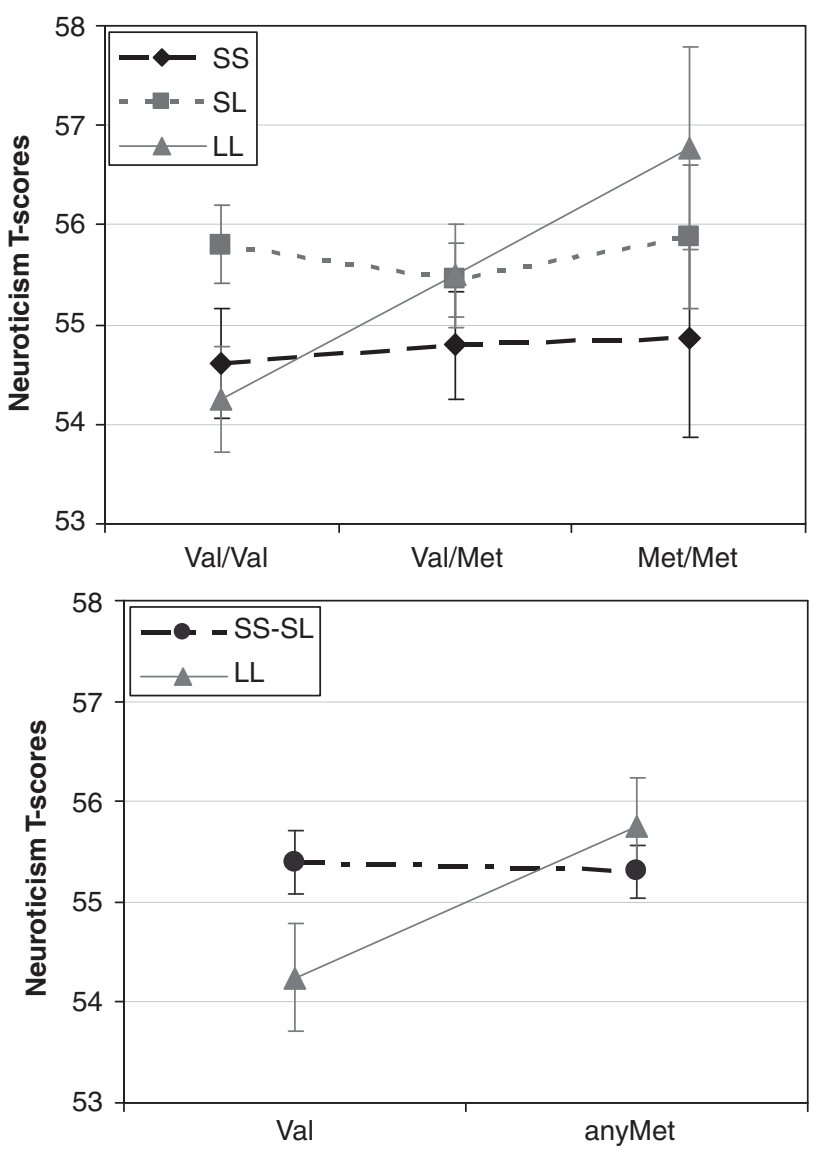

Figure 2 Interaction of BDNF Val66Met and 5-HTTLPR on NEO-PI-R neuroticism scores in the SardiNIA sample $(N=2333)$. Among the carriers of the 5-HTTLPR LL genotype, individuals with one or two copies of the BDNF Met variant tend to score higher on neuroticism ( $p=0.005)$. Among individuals with the 5-HTTLPR S variant, there are no significant differences in neuroticism scores between BDNF genotypes $(p=0.98)$.

2008), we found an association between BDNF and extraversion in a replication sample from SardiNIA and one from the United States. The effects were small but consistent across samples, with the Met carriers scoring roughly $0.2 \mathrm{SD}$ lower on extraversion compared with individuals with the Val/Val genotype. Previous research has shown that the BDNF Met variant is linked to poor intracellular trafficking, decreased BDNF secretion, and abnormal hippocampus structure and function (Bueller et al, 2006; Egan et al, 2003). To this literature we add that Met carriers are also more likely to be introverted. This is of interest from a clinical perspective, as introverts tend to be socially withdrawn and more often experience anhedonia and dysthymic/depressed mood (Bienvenu et al, 2004; Clark et al, 1994; Jylha et al, 2009; Watson et al, 2005). These findings can provide new insights into animal and clinical studies that examine the effects of BDNF on mood, cognition, and behaviors.

The association between introversion and the Met variant is consistent with the idea that genetic factors contribute to the personality differences observed across populations. Indeed, cross-cultural studies of personality have consistently found that individuals from Asian populations tend to score slightly lower on extraversion compared with individuals of European ancestry (McCrae and Terracciano, 2005b; McCrae et al, in press; Schmitt et al, 2007). The rs6265 (Val66Met) allele frequencies differ across populations, with the A (Met) allele estimated to be about $20 \%$ among Europeans but about $40 \%$ among Asians (Itoh et al, 2004; Tochigi et al, 2006). As such, the higher frequency of the A (Met) allele among Asian populations may contribute to their lower scores on extraversion. Of course, cultural factors underlie these cross-cultural differences, but if confirmed in additional large samples, this association could be, to our knowledge, the first ascertained genetic variant that contributes to personality differences across populations.

We found no evidence of an association between BDNF Val66Met and neuroticism in two independent samples and in the meta-analysis inclusive of the published literature (see Figure 2). Although there is no evidence for a main effect on neuroticism, the effect of BDNF may depend, in part, on interactions with either environmental factors (gene $x$ environment) or other genetic variants (gene $\times$ gene).

Animal models and clinical studies suggest that BDNF and the serotonin system are interdependent (Henningsson et al, 2009; Martinowich and Lu, 2008; Mattson et al, 2004), which has generated the hypothesis that the two genes interact (Pezawas et al, 2008; Rybakowski et al, 2007; Wichers et al, 2008). We tested for epistatic effects of Val66Met and 5-HTTLPR on neuroticism using the largest sample examined so far for depression-related outcomes. Despite the lack of main effects for BDNF Val66Met (see Figure 1) and 5-HTTLPR on neuroticism (Munafo et al, 2008; Terracciano et al, 2009a; Willis-Owen et al, 2005b), we found an interaction between these two genetic variants. Among the carriers of the 5-HTTLPR LL genotype, individuals with the BDNF Met variant scored higher on neuroticism compared with those with the Val variant. No differences were observed among 5-HTTLPR S carriers. This effect differs from those reported in previous smaller studies (Pezawas et al, 2008; Rybakowski et al, 2007; Wichers et al, 2008). Nevertheless, higher scores on neuroticism seem consistent with a synergistic effect of the BDNF Met variant, which reduces BDNF secretion and leads to anxiety-related behaviors in mice (Chen et al, 2006; Egan et al, 2003; Matsuo et al, 2009), and with the 5-HTTLPR LL genotype, which is associated with a higher rate of serotonin re-uptake from the synaptic cleft and supposedly to serotonin depletion (Arbelle et al, 2003; Lesch et al, 1996; Terracciano et al, 2009a). Given our promising findings and the intuitive appeal of an interaction between variants that reduce the circulating level of BDNF and serotonin, larger studies should attempt to confirm this epistatic effect on this major risk factor for depression.

This study advances our understanding of the influence of BDNF and 5-HTT on neuroticism and extraversion, the two personality dimensions with the strongest links to emotional processing. Identifying the genetic underpinnings of neuroticism and extraversion can help elucidate the biological pathways that contribute to psychiatric disorders with strong affective components.

\section{ACKNOWLEDGEMENTS}

We thank the individuals who participated in this study; The SardiNIA team thanks Monsignore Piseddu (Bishop of 
Ogliastra), the mayors of the four Sardinian towns (Lanusei, Ilbono, Arzana, and Elini), and the head of the Public Health Unit ASL4 for cooperation. We thank Professor Antonio Cao for his leadership of the SardiNIA project. This research was supported entirely by the Intramural Research Program of the NIH, National Institute on Aging.

\section{DISCLOSURE}

Paul T. Costa, Jr receives royalties from the Revised NEO Personality Inventory. The authors declare no other conflict of interest.

\section{REFERENCES}

Arbelle S, Benjamin J, Golin M, Kremer I, Belmaker RH, Ebstein RP (2003). Relation of shyness in grade school children to the genotype for the long form of the serotonin transporter promoter region polymorphism. Am J Psychiatry 160: 671-676.

Bienvenu OJ, Samuels JF, Costa PT, Reti IM, Eaton WW, Nestadt G (2004). Anxiety depressive disorders the five-factor model of personality: a higher- lower-order personality trait investigation in a community sample. Depress Anxiety 20: 92-97.

Bouchard TJ, Loehlin JC (2001). Genes evolution, personality. Behav Genet 31: 243-273.

Bueller JA, Aftab M, Sen S, Gomez-Hassan D, Burmeister M, Zubieta JK (2006). BDNF Val66Met allele is associated with reduced hippocampal volume in healthy subjects. Biol Psychiatry 59: $812-815$.

Carvalho AL, Caldeira MV, Santos SD, Duarte CB (2008). Role of the brain-derived neurotrophic factor at glutamatergic synapses. Br J Pharmacol 153(Suppl 1): S310-S324.

Chen B, Dowlatshahi D, MacQueen GM, Wang JF, Young LT (2001). Increased hippocampal BDNF immunoreactivity in subjects treated with antidepressant medication. Biol Psychiatry 50: $260-265$.

Chen WM, Abecasis GR (2007). Family-based association tests for genomewide association scans. Am J Hum Genet 81: 913-926.

Chen ZY, Jing D, Bath KG, Ieraci A, Khan T, Siao CJ et al (2006). Genetic variant BDNF (Val66Met) polymorphism alters anxietyrelated behavior. Science 314: 140-143.

Clark LA, Watson D, Mineka S (1994). Temperament, personality, and the mood and anxiety disorders. J Abnorm Psychol 103: 103-116.

Costa Jr PT, McCrae RR (1980). Influence of extraversion neuroticism on subjective well-being: happy unhappy people. J Pers Soc Psychol 38: 668-678.

Costa Jr PT, McCrae RR (1992). Revised NEO Personality Inventory (NEO-PI-R) and NEO Five-Factor Inventory (NEO-FFI) Professional Manual. Psychological Assessment Resources: Odessa, FL.

Costa Jr PT, Terracciano A, McCrae RR (2001). Gender differences in personality traits across cultures: robust and surprising findings. J Pers Soc Psychol 81: 322-331.

Costa Jr PT, Terracciano A, Uda M, Vacca L, Mameli C, Pilia G et al (2007). Personality traits in Sardinia: testing founder population effects on trait means and variances. Behav Genet 37: 376-387.

Cotman CW, Berchtold NC (2002). Exercise: a behavioral intervention to enhance brain health and plasticity. Trends Neurosci 25: 295-301.

Devlin B, Roeder K (1999). Genomic control for association studies. Biometrics 55: 997-1004.

Egan MF, Kojima M, Callicott JH, Goldberg TE, Kolachana BS, Bertolino A et al (2003). The BDNF val66met polymorphism affects activity-dependent secretion of BDNF and human memory and hippocampal function. Cell 112: 257-269.
Frustaci A, Pozzi G, Gianfagna F, Manzoli L, Boccia S (2008). Metaanalysis of the brain-derived neurotrophic factor gene (BDNF) Val66Met polymorphism in anxiety disorders and anxietyrelated personality traits. Neuropsychobiology 58: 163-170.

Gatt JM, Nemeroff CB, Dobson-Stone C, Paul RH, Bryant RA, Schofield PR et al (2009). Interactions between BDNF Val66Met polymorphism and early life stress predict brain and arousal pathways to syndromal depression and anxiety. Mol Psychiatry 14: 681-695.

Gratacos M, Gonzalez JR, Mercader JM, de Cid R, Urretavizcaya M, Estivill X (2007). Brain-derived neurotrophic factor Val66Met and psychiatric disorders: meta-analysis of case-control studies confirm association to substance-related disorders, eating disorders, and schizophrenia. Biol Psychiatry 61: 911-922.

Green EK, Raybould R, Macgregor S, Hyde S, Young AH, O'Donovan MC et al (2006). Genetic variation of brain-derived neurotrophic factor (BDNF) in bipolar disorder: case-control study of over 3000 individuals from the UK. Br J Psychiatry 188: 21-25.

Gunthert KC, Conner TS, Armeli S, Tennen H, Covault J, Kranzler HR (2007). Serotonin transporter gene polymorphism (5HTTLPR) and anxiety reactivity in daily life: a daily process approach to gene-environment interaction. Psychosom Med 69: $762-768$.

Henningsson S, Borg J, Lundberg J, Bah J, Lindstrom M, Ryding E et al (2009). Genetic variation in brain-derived neurotrophic factor is associated with serotonin transporter but not serotonin1a receptor availability in men. Biol Psychiatry 66: 477-885.

Hunnerkopf R, Strobel A, Gutknecht L, Brocke B, Lesch KP (2007). Interaction between BDNF Val66Met and dopamine transporter gene variation influences anxiety-related traits. Neuropsychopharmacology 32: 2552-2560.

Itoh K, Hashimoto K, Kumakiri C, Shimizu E, Iyo M (2004). Association between brain-derived neurotrophic factor $196 \mathrm{G} / \mathrm{A}$ polymorphism and personality traits in healthy subjects. Am J Med Genet B Neuropsychiatr Genet 124B: 61-63.

Jylha P, Melartin T, Rytsala H, Isometsä E (2009). Neuroticism, introversion, and major depressive disorder - traits, states, or scars? Depress Anxiety 26: 325-334.

Kendler KS, Gatz M, Gardner CO, Pedersen NL (2006). Personality and major depression: a Swedish longitudinal, population-based twin study. Arch Gen Psychiatry 63: 1113-1120.

Kim JM, Stewart R, Kim SW, Yang SJ, Shin IS, Kim YH et al (2007). Interactions between life stressors and susceptibility genes (5HTTLPR and BDNF) on depression in Korean elders. Biol Psychiatry 62: 423-428.

Lang UE, Hellweg R, Kalus P, Bajbouj M, Lenzen KP, Sander T et al (2005). Association of a functional BDNF polymorphism and anxiety-related personality traits. Psychopharmacology (Berl) 180: 95-99.

Lesch KP, Bengel D, Heils A, Sabol SZ, Greenberg BD, Petri S et al (1996). Association of anxiety-related traits with a polymorphism in the serotonin transporter gene regulatory region. Science 274: 1527-1531.

Mamounas LA, Blue ME, Siuciak JA, Altar CA (1995). Brainderived neurotrophic factor promotes the survival and sprouting of serotonergic axons in rat brain. J Neurosci 15: 7929-7939.

Martinowich K, Lu B (2008). Interaction between BDNF and serotonin: role in mood disorders. Neuropsychopharmacology 33: 73-83.

Matsuo K, Walss-Bass C, Nery FG, Nicoletti MA, Hatch JP, Frey BN et al (2009). Neuronal correlates of brain-derived neurotrophic factor Val66Met polymorphism and morphometric abnormalities in bipolar disorder. Neuropsychopharmacology 34: 19041913.

Mattson MP, Maudsley S, Martin B (2004). BDNF and 5-HT: a dynamic duo in age-related neuronal plasticity and neurodegenerative disorders. Trends Neurosci 27: 589-594. 
McCrae RR, Terracciano A, 78 Members of the Personality Profiles of Cultures Project (2005a). Universal features of personality traits from the observer's perspective: data from 50 cultures. J Pers Soc Psychol 88: 547-561.

McCrae RR, Terracciano A, 79 Member of the Personality Profiles of Cultures Project (2005b). Personality profiles of cultures: aggregate personality traits. J Pers Soc Psychol 89: 407-425.

McCrae RR, Terracciano A, De Fruyt F, De Bolle M, Gelfand MJ, Costa PTJ, et al. The validity and structure of culture-level personality scores: data from ratings of young adolescents. J Pers. (in press).

Mossner R, Daniel S, Albert D, Heils A, Okladnova O, Schmitt A, et al (2000). Serotonin transporter function is modulated by brain-derived neurotrophic factor (BDNF) but not nerve growth factor (NGF). Neurochem Int 36: 197-202.

Muglia P, Tozzi F, Galwey NW, Francks C, Upmanyu R, Kong XQ et al (2008). Genome-wide association study of recurrent major depressive disorder in two European case-control cohorts. Mol Psychiatry; e-pub ahead of print 28 December 2008.

Munafo MR, Freimer NB, Ng W, Ophoff R, Veijola J, Miettunen J et al (2008). 5-HTTLPR genotype and anxiety-related personality traits: a meta-analysis and new data. Am J Med Genet B Neuropsychiatr Genet 150B: 271-281.

Nibuya M, Morinobu S, Duman RS (1995). Regulation of BDNF and trkB mRNA in rat brain by chronic electroconvulsive seizure and antidepressant drug treatments. J Neurosci 15: 7539-7547.

Pezawas L, Meyer-Lindenberg A, Goldman AL, Verchinski BA, Chen G, Kolachana BS et al (2008). Evidence of biologic epistasis between BDNF and SLC6A4 and implications for depression. Mol Psychiatry 13: 709-716.

Pilia G, Chen WM, Scuteri A, Orrú M, Albai G, Dei M et al (2006). Heritability of cardiovascular and personality traits in 6148 Sardinians. PloS Genetics 2: e132.

Price AL, Patterson NJ, Plenge RM, Weinblatt ME, Shadick NA, Reich D (2006). Principal components analysis corrects for stratification in genome-wide association studies. Nat Genet 38: 904-909.

Rybakowski JK (2008). BDNF gene: functional Val66Met polymorphism in mood disorders and schizophrenia. Pharmacogenomics 9: 1589-1593.

Rybakowski JK, Suwalska A, Skibinska M, Dmitrzak-Weglarz M, Leszczynska-Rodziewicz A, Hauser J (2007). Response to lithium prophylaxis: interaction between serotonin transporter and BDNF genes. Am J Med Genet B Neuropsychiatr Genet 144B: 820-823.

Schmitt DP, Allik J, McCrae RR, Benet-Martinez V (2007). The geographic distribution of big five personality traits: patterns and profiles of human self-description across 56 nations. J Cross Cult Psychol 38: 173-212.

Sen S, Duman R, Sanacora G (2008). Serum brain-derived neurotrophic factor, depression, and antidepressant medications: meta-analyses and implications. Biol Psychiatry 64: 527532.

Sen S, Nesse RM, Stoltenberg SF, Li S, Gleiberman L, Chakravarti A et al (2003). A BDNF coding variant is associated with the NEO personality inventory domain neuroticism, a risk factor for depression. Neuropsychopharmacology 28: 397-401.

Siuciak JA, Lewis DR, Wiegand SJ, Lindsay RM (1996). Antidepressant-like effect of brain-derived neurotrophic factor (BDNF). Pharmacol Biochem Behav 56: 131-137.

Sklar P, Gabriel SB, McInnis MG, Bennett P, Lim YM, Tsan G et al (2002). Family-based association study of 76 candidate genes in bipolar disorder: BDNF is a potential risk locus. Mol Psychiatry 7: 579-593.

Terracciano A (2003). The Italian version of the NEO PI-R: conceptual and empirical support for the use of targeted rotation. Pers Individ Dif 35: 1859-1872.

Terracciano A, Balaci L, Thayer J, Scally M, Kokinos S, Ferrucci L et al (2009a). Variants of the serotonin transporter gene and
NEO-PI-R Neuroticism: no association in the BLSA and SardiNIA samples. Am J Med Genet B Neuropsychiatr Genet 150B: 1070-1077.

Terracciano A, Costa PTJ, McCrae RR (2006). Personality plasticity after age 30. Pers Soc Psychol Bull 32: 999-1009.

Terracciano A, McCrae RR, Brant LJ, Costa Jr PT (2005). Hierarchical linear modeling analyses of NEO-PI-R scales in the Baltimore Longitudinal Study of Aging. Psychol Aging 20: 493-506.

Terracciano A, McCrae RR, Costa Jr PT (in press) Intra-individual change in personality stability and age. J R Pers; doi:10.1016/ j.jrp.2009.1009.1006.

Terracciano A, Sanna S, Uda M, Deiana B, Usala G, Busonero F et al (2008). Genome-wide association scan for five major dimensions of personality. Mol Psychiatry; e-pub ahead of print 28 October 2008, doi: 10.1038/mp.2008.1113.

Terracciano A, Sutin AR, McCrae RR, Deiana B, Ferrucci L, Schlessinger D et al (2009b). Facets of personality linked to underweight and overweight. Psychosom Med 71: 682-689.

Tochigi M, Otowa T, Suga M, Rogers M, Minato T, Yamasue H et al (2006). No evidence for an association between the BDNF Val66Met polymorphism and schizophrenia or personality traits. Schizophr Res 87: 45-47.

van den Oord EJCG, Kuo P-H, Hartmann AM, Webb BT, Moller HJ, Hettema JM et al (2008). Genomewide association analysis followed by a replication study implicates a novel candidate gene for neuroticism. Arch Gen Psychiatry 65: 1062-1071.

Wachleski C, Blaya C, Salum GA, Vargas V, Leistner-Segal S, Manfro GG (2008). Lack of association between the serotonin transporter promoter polymorphism (5-HTTLPR) and personality traits in asymptomatic patients with panic disorder. Neurosci Lett 431: 173-178.

Watson D, Clark LA (1992). On traits and temperament: general and specific factors of emotional experience and their relation to the five-factor model. $J$ Pers 60: 441-476.

Watson D, Gamez W, Simms LJ (2005). Basic dimensions of temperament and their relation to anxiety and depression: a symptom-based perspective. J Res Pers 39: 46-66.

Wendland JR, Kruse MR, Cromer KR, Murphy DL (2007). A large case-control study of common functional SLC6A4 and BDNF variants in obsessive-compulsive disorder. Neuropsychopharmacology 32: 2543-2551.

Wichers M, Kenis G, Jacobs N, Mengelers R, Derom C, Vlietinck R

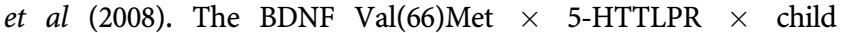
adversity interaction and depressive symptoms: an attempt at replication. Am J Med Genet B Neuropsychiatr Genet 147B: 120-123. Willis-Owen SA, Fullerton J, Surtees PG, Wainwright NW, Miller S, Flint J (2005a). The Val66Met coding variant of the brainderived neurotrophic factor (BDNF) gene does not contribute toward variation in the personality trait neuroticism. Biol Psychiatry 58: 738-742.

Willis-Owen SA, Turri MG, Munafo MR, Surtees PG, Wainwright NW, Brixey RD et al (2005b). The serotonin transporter length polymorphism, neuroticism, and depression: a comprehensive assessment of association. Biol Psychiatry 58: 451-456.

Wray NR, James MR, Gordon SD, Dumenil T, Ryan L, Coventry WL et al (2009). Accurate, large-scale genotyping of 5HTTLPR and flanking single nucleotide polymorphisms in an association study of depression, anxiety, and personality measures. Biol Psychiatry 66: 468-476.

Wray NR, James MR, Handoko HY, Dumenil T, Lind PA, Montgomery GW et al (2008). Association study of candidate variants from brain-derived neurotrophic factor and dystrobrevin-binding protein 1 with neuroticism, anxiety, and depression. Psychiatr Genet 18: 219-225.

Zhou J, Li L, Tang S, Cao X, Li Z, Li W et al (2008). Effects of serotonin depletion on the hippocampal GR/MR and BDNF expression during the stress adaptation. Behav Brain Res 195: $129-138$ 